\title{
Physiology and cell biology of acupuncture observed in calcium signaling activated by acoustic shear wave
}

\author{
Geng Li $\cdot$ Jie-Ming Liang $\cdot$ Pei-Wen Li $\cdot$ Xiaoqiang Yao $\cdot$ Peter Zhong Pei $\cdot$ Wei Li $\cdot$ \\ Qi-Hua He • Xifei Yang • Queenie C. C. Chan • Paul Y. S. Cheung • Qi Yuan Ma • \\ Siu Kam Lam • Patrick Y. C. Cheng • Edward S. Yang
}

Received: 7 June 2011 /Revised: 28 June 2011 / Accepted: 29 June 2011 /Published online: 28 July 2011

(C) The Author(s) 2011. This article is published with open access at Springerlink.com

\begin{abstract}
This article presents a novel model of acupuncture physiology based on cellular calcium activation by an acoustic shear wave (ASW) generated by the mechanical movement of the needle. An acupuncture needle was driven by a piezoelectric transducer at $100 \mathrm{~Hz}$ or below, and the ASW in human calf was imaged by magnetic resonance elastography. At the cell level, the ASW activated intracellular $\mathrm{Ca}^{2+}$ transients and oscillations in fibroblasts and endothelial, ventricular
\end{abstract}

Geng Li and Jie-Ming Liang contributed equally to this work.

G. Li $\cdot$ Q. Y. Ma $\cdot$ E. S. Yang

Time Medical Systems,

No. 2, Science Park West Avenue,

Hong Kong, China

\section{J.-M. Liang}

Biomedical Engineering, The University of Hong Kong,

Hong Kong, China

P. Y. S. Cheung $\cdot$ S. K. Lam • P. Y. C. Cheng • E. S. Yang

The University of Hong Kong,

Hong Kong, China

P.-W. Li • E. S. Yang

EE Department, National Central University,

Chungli, Taiwan

\section{Yao}

School of Biomedical Sciences,

Chinese University of Hong Kong,

Hong Kong, China myocytes and neuronal PC-12 cells along with frequency-amplitude tuning and memory capabilities. Monitoring in vivo mammalian experiments with ASW, enhancement of endorphin in blood plasma and blocking by $\mathrm{Gd}^{3+}$ were observed; and increased $\mathrm{Ca}^{2+}$ fluorescence in mouse hind leg muscle was imaged by two-photon microscopy. In contrast with traditional acupuncture models, the signal source is derived from the total acoustic energy. ASW signaling makes use of the anisotropy of elasticity of 
tissues as its waveguides for transmission and that cell activation is not based on the nervous system.

Keywords Acupuncture $\cdot$ Acoustic wave $\cdot \mathrm{MRE} \cdot \mathrm{Ca}^{2+}$ ion channel $\cdot$ Human $\cdot$ Mice $\cdot$ Fibroblast $\cdot$ Endothelial cells

\section{Abbreviations \\ ASW Acoustic shear wave \\ MRE Magnetic resonance elastography}

\section{Introduction}

Acupuncture (Latin for needle prick) is an ancient Chinese art of healing. Along with it is a system of tracks called meridians by the practitioners but invisible anatomically. The great biochemist-turned-historian Joseph Needham described it intuitively as "a system of channels for transporting energy and fundamentally circulatory in nature to all tissues round the body" [15]. Although this medical practice has been in existence for three millennia, its therapeutic purpose has been considered seriously in the West only in the last 30 years [20]. The pertinent question is whether this art of healing can be turned into science, making the experiments observable, reproducible, and conformable to the current knowledge of physiology and cell biology.

Recently, acupuncture analgesia received strong support from clinical studies in Germany. Randomized largescale clinical trials have demonstrated its effectiveness in migraine [4, 14], osteoarthritis [23], and chronic knee and back pain [7]. Various techniques have been tried to elucidate the basic mechanism of acupuncture. These include thermal imaging, ultrasound, protoninduced X-ray emission, low resistance path tracing, radioactive tracing, and trigger point correlation among others [20]. Making use of functional magnetic resonance imaging (MRI), activation sites in the brain have been reported in tests with acupuncture [11, 13]. However, the nature of the acupuncture signal and its transmission remain elusive.

Our approach to unravel the complexity of acupuncture was to get down to the most elementary principles. We started by formulating the mechanical movement of the needle of the acupuncturist as an acoustic shear wave (ASW) such that its function is mathematically well defined. The model was tested in human calf muscle and observed by using a method called magnetic resonance elastography (MRE) [16]. In MRI, a standard practice of imaging the slow movement of molecules in blood flow is known as the MR angiography. MRE is based on the same principle except that an ASW is used and the molecules observed are in tissues and muscles. The acoustic signal is generated by a piezoelectric transducer and coupled to the tissues of interest. A pulse sequence using phase-locked cyclic motion-sensitizing gradients is employed to encode the motion as phases in the MR images. By accumulating phases during several wave cycles, the signal can depict cyclic motions with an amplitude less than $100 \mathrm{~nm}$ [16]. Instead of modeling the jerking and irregular motions of the typical needle, we used a sinusoid so that the result and interpretation were simplified. Human subjects were employed.

To observe the cellular response to ASW, cells were put in a Petri dish in a high-viscosity methyl cellulose solution. This special arrangement is necessary to mimic the tissues and provide proper acoustic wave coupling not available with water. An acupuncture needle driven by a piezoelectric element was applied to the gel to produce the ASW. The ASW was imaged by MRE and the activities of the cells were observed under a confocal microscope. Spatial and temporal responses through $\mathrm{Ca}^{2+}$ activation were recorded for fibroblast, endothelial, cardiac myocytes, and neuronal PC-12 cells.

To link the calcium activities from cell lines to animals, $\mathrm{Ca}^{2+}$ fluorescence was observed in vivo using a two-photon laser imaging technique. Additional support of calcium activation was obtained by measurement of endorphin in blood plasma and $\mathrm{Gd}^{3+}$ blocking. The series of experiments were designed to show that the self-consistency of the proposed model has no internal conflicts. No experiment on pain suppression was carried out.

\section{Methods}

\section{Mechanical measurement}

In studying acupuncture, an important and frequently overlooked procedure is the manual needle manipulation performed by acupuncturists after needle insertion. The needle manipulations are typically a series of rapid bidirectional rotation or up-and-down piston movements. Spatially mapped and quantified by ultrasound, the needle stimulus is visualized to wind the tissue and cause tissue displacements of up to $100 \mu \mathrm{m}$ [12]. In our experiment, the displacement produced by the moving needle was directly measured and visualized with MRE. MRE with a piezoelectric-driven needle driver $[3,16]$ was used to observe the generation and transmission of the ASW in clinical acupuncture in human subjects. The acupuncture needle was inserted at the acupoint GB 35 (Fig. 1a) located at the calf muscle and adjacent nonacupoint located $1 \mathrm{~cm}$ laterally in a GE 1.5-T scanner. The waves induced by the vibrating needle (Fig. 1b) were 

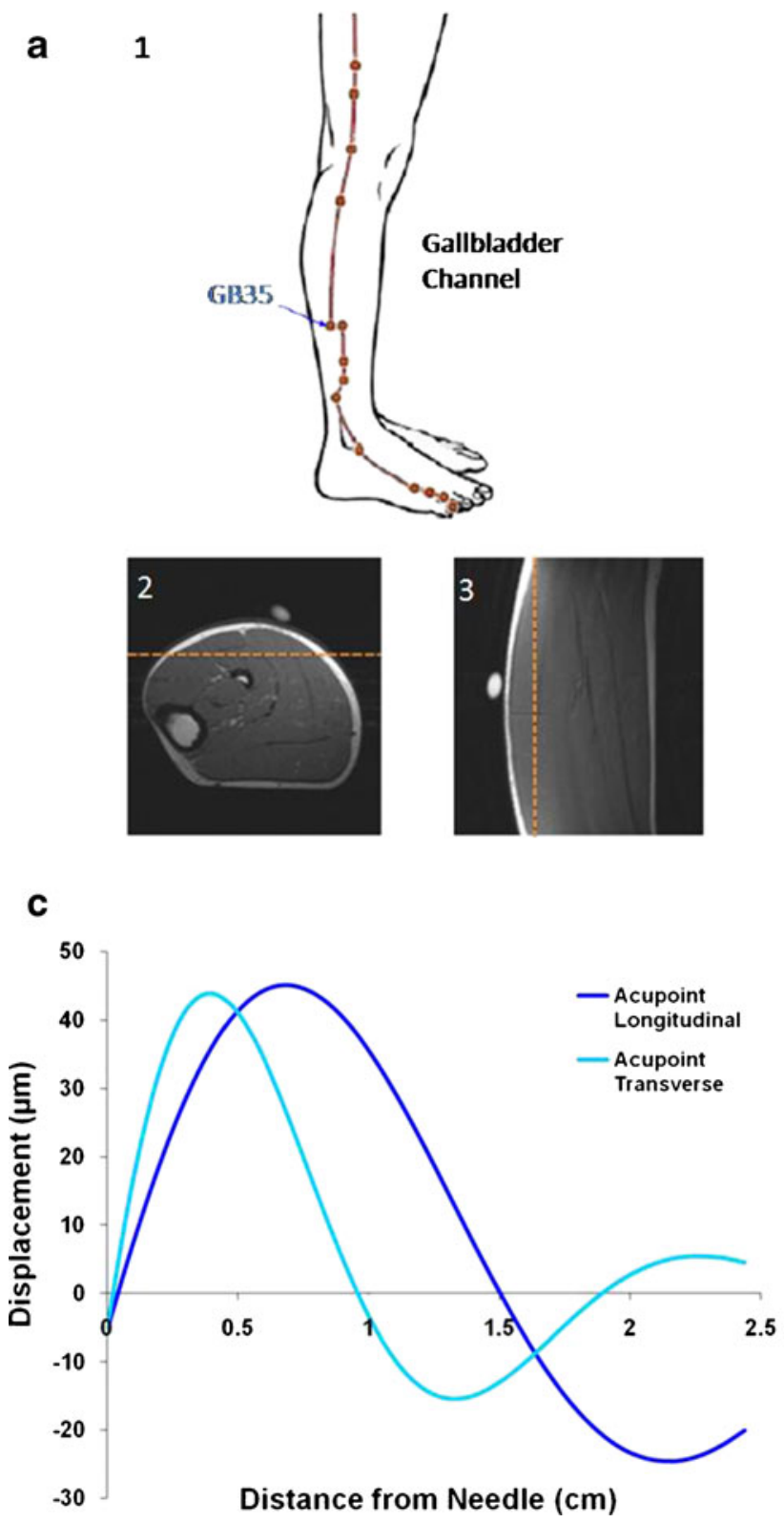

Fig. 1 Study of acupuncture mechanism based on acoustic wave signaling. a Location of acupoint GB 35 in the gallbladder channel. T1 images showing the slice orientation of the MRE scan. b Coronal T1-weighted images, wave images in transverse direction, and wave images in longitudinal direction with needle at acupoint (blue open

measured with a phase-locked motion-sensitizing field gradient echo MR sequence. The direction of the motionsensitizing field gradients was collinear with the longitudinal motion of the driver and perpendicular to the wave propagation direction. Figure 1c shows an example of the fitted line profile data to damped sinusoid model in the form of $A(x)=A_{0} \cdot \sin (k x) \cdot \exp (-x / d)$ at the acupoint, where $x$ is the distance away from the needle activation, $A_{\mathrm{o}}$ is the initial amplitude of the field at the needle, $k$ is the wave number equal to $2 \pi / \lambda$ and $\lambda$ is the wavelength, and $d$ is the b

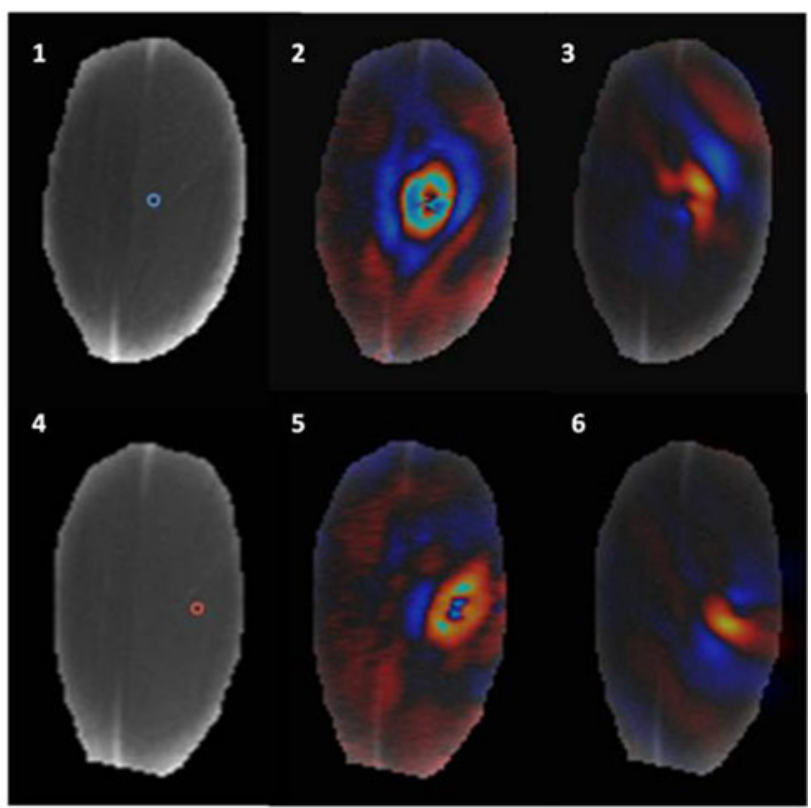

d

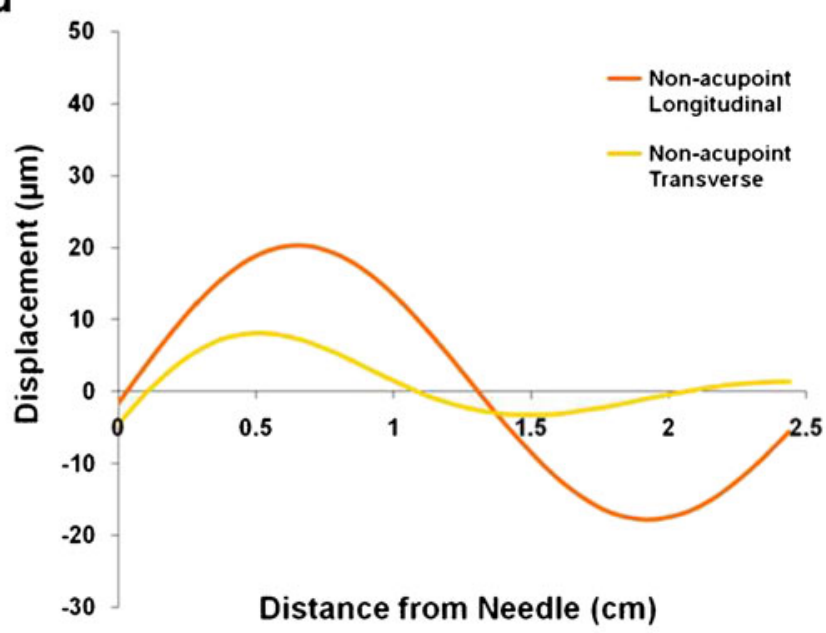

circle, 1-3), and adjacent non-acupoint (red open circle, 4-6). c Wave propagation profiles in longitudinal and transverse direction at acupoint. d Wave propagation profiles in longitudinal and transverse directions at non-acupoint

delay or attenuation length. The shear stiffness is calculated using Eq. $1[1,10]$ and the anisotropic index was calculated using Eq. 2 [19].

Cell culture

Mouse embryonic fibroblast cell line NIH 3T3 and murine microvascular endothelial cell line $\mathrm{H} 5 \mathrm{~V}$ were maintained at $37^{\circ} \mathrm{C}$ in a $5 \% \mathrm{CO}_{2}$ humidified incubator in Dulbecco's modified Eagle's medium (Gibco) supplemented with $10 \%$ 
fetal bovine serum (Gibco), $100 \mathrm{U} / \mathrm{ml}$ penicillin, and $100 \mu \mathrm{g} / \mathrm{ml}$ streptomycin.

\section{Calcium imaging}

Cells were grown to $80 \%$ confluence prior to calcium imaging. The fluorescent $\mathrm{Ca}^{2+}$ indicator dye is Calcium Green-1 for NIH 3T3 and Fluo-4/AM for H5V. The free cytosolic $\mathrm{Ca}^{2+}$ concentration in NIH $3 \mathrm{~T} 3$ and $\mathrm{H} 5 \mathrm{~V}$ was recorded by the inverted laser-scanning confocal microscope (Olympus FluoView FV1000). Cells were bathed in 2\% methyl cellulose solution with Hank's buffered salt solution (Gibco) as basal medium for confocal imaging. The same piezoelectric needle driver was applied to generate acoustic waves in the methyl cellulose medium.

In vivo two-photon calcium imaging

With regard to animal ethics, approval was obtained through Peking/Beijing University (PKU) IACUC for live animal acupuncture studies under approval ID IMM-ChengHP-14. The hind limb muscle of 8-week-old C57BL/6N mice was transfected with GCaMP 2 cDNA by electroporation. In brief, $40 \mu \mathrm{g}$ of expression plasmid was injected, followed by controlled electric pulses ( $30 \mathrm{~V} / 20 \mathrm{~ms}$ duration) delivered by needle electrodes inserted into the skeletal muscle. One to two weeks after transfection, the operated animal was anesthetized, shaved, and mounted for observation. The acupuncture needle was inserted into the hind limb muscle at the acupoint ST36 and the acupuncture needle was driven by a piezoelectric bending element (D220-A4-503YB, Piezo Systems, Inc.) at $1 \mathrm{~Hz}$. A sham acupuncture needle was inserted into the hind limb muscle without any stimulation. $\mathrm{Ca}^{2+}$ signal was monitored by the Leica TCS SP5 twophoton laser-scanning microscope system coupled to an upright microscope (CFS, Leica). Two-photon fluorescence images were acquired through a $\times 20$ water immersion objective (HCX, APO, $\times 1.20 / 1.00 \mathrm{~W}$, Leica) using $488 \mathrm{~nm}$ light for fluorescence excitation (argon laser).

\section{$\beta$-Endorphin immunoassay}

Four-week-old C57BL/6N mice were kept in a specially designed holder with their hind legs and tails exposed during acupuncture. An acupuncture needle (Hwato) $0.4 \mathrm{~mm}$ in diameter and $50 \mathrm{~mm}$ in length was inserted into the right hind leg, between the tibia and fibula, approximately $5 \mathrm{~mm}$ lateral to the anterior tubercle of the tibia. The acupuncture needle was driven by a piezoelectric bending element (D220-A4-503YB, Piezo Systems, Inc.) at $1 \mathrm{~Hz}$. After receiving acupuncture treatment for $40 \mathrm{~min}$, animals were sacrificed. Blood samples were harvested from the left ventricle of the heart. Plasma was separated by centrifuging samples at $1,000 \times g$ for 15 min at $4^{\circ} \mathrm{C}$ and diluted $1: 10$ in a sample diluted before assay. The levels of plasma $\beta$-endorphin were measured using a mouse $\beta$-endorphin ELISA kit from USCN Life Science.

\section{Magnetic resonance elastography}

With regard to human ethics, institutional review board approval was obtained through the University of Hong Kong under approval ID \#UW04-128T/450. Written informed consent was obtained for all volunteers. Twelve healthy male volunteers aged $24.83 \pm 1.95$ years, with weight of $63.83 \pm 6.81 \mathrm{~kg}$ and height of $170.25 \pm 7.57 \mathrm{~cm}$, underwent the acupuncture study by MRE [16]. Insertion of the acupuncture needle at the acupoint GB 35 and adjacent non-acupoint during in vivo MRE scans was performed in humans at the right leg. The non-acupoint was located $1 \mathrm{~cm}$ lateral to the acupoint GB 35. The needle driver consisted of a piezoelectric bending element (D220-A4-503YB, Piezo Systems, Inc.). The needles (Hwato) were $0.4 \mathrm{~mm}$ in diameter, $50 \mathrm{~mm}$ long, and made of sterilized pure silver, which were disposed after use. Mechanical excitation was applied at the thigh muscle by the vertical movements of the needle. The MRE study was performed in a 1.5-T MRI scanner (Signa Horizon Echo Speed with version 5.51 software, General Electric Medical Systems, Milwaukee, WI, USA). The waves induced by the needle were measured with a phase-locked motion-sensitized gradient echo MR sequence. Typical data acquisition parameters were TR, $150 \mathrm{~ms}$; TE, $50 \mathrm{~ms}$; flip angle, $30^{\circ}$; acquisition matrix, 256 $\times 64$; field of view (FOV), $24 \mathrm{~cm}$; and acquisition time, $115.2 \mathrm{~s}$ per slice. The frequency of mechanical excitation was fixed at $100 \mathrm{~Hz}$. The number of motion-sensitizing gradients used was 2 at $100 \mathrm{~Hz}$. Six dynamics were obtained by changing the phase offset between the mechanical excitation and the motionsensitizing gradient to obtain the image of propagation of the shear waves. We define longitudinal as the direction along the muscle fiber and transverse as the direction perpendicular to the muscle fiber.

\section{Phantom experiments}

The same needle driver described above was used to generate propagating waves through a $2 \%$ methyl cellulose medium (4,000 cPs viscosity, Sigma) in a chamber of $100 \mathrm{~mm}$ diameter and $20 \mathrm{~mm}$ height. MRE was applied to visualize propagating shear waves. Wave images were obtained on a Philips Intera Achieva 3T system with SENSE Flex-M coil. The FOV was $90 \mathrm{~mm}$ with an isotropic voxel size of $1.4 \mathrm{~mm}$ in all three directions. The mechanical excitation frequency was $80 \mathrm{~Hz}$. A Piezo- 
Acoustic Transducer Model in COMSOL Multiphysics 3.4 (http://www.comsol.com/showroom/gallery/1477/) was used to simulate the acoustic pressure distribution within methyl cellulose solution according to manufacturer's instructions.

\section{Statistical analyses}

Statistical data were reported as means \pm SEM. Significance was tested by analysis of variance or Student's $t$ test. A $p$ value less than 0.05 was considered statistically significant.

\section{Basic formulae in acoustics}

The shear stiffness is calculated using the following equation:

$\mu=\rho f^{2} \lambda^{2}$

where $\rho$ is the density of the material, $f$ is the excitation frequency, and $\lambda$ is the wavelength determined from the wave images. MRE data were processed using a damped sinusoid model. A line profile was drawn along the wave propagation direction and fitted into the damped sinusoid model to estimate the wavelength. The density $\rho$ is assumed to be $1.1 \mathrm{~g} / \mathrm{cm}^{3}$ for muscle tissue in the literature [5].

Anisotropic index was calculated using the equation:

$\tau=\mu_{/ /}-\mu_{\perp}$

where $\mu_{/ /}$is the shear stiffness measured along the muscle fiber direction and $\mu_{\perp}$ is the shear stiffness measured in the perpendicular direction.

\section{Results}

Based on the procedure discussed in relation to Fig. 1a-c, we are in a position to study the nature of acoustic wave propagation in the human muscle. The significant difference of the longitudinal and transverse components came from the anisotropy of the muscle elasticity without any reflected waves. The amplitude decayed to a tenth of its initial value in 6.18 and $2.43 \mathrm{~cm}$ along the longitudinal and transverse direction, respectively, suggesting the corresponding decay length of 2.684 and $1.055 \mathrm{~cm}$. The fitted line profile of the longitudinal component at nonacupoint is shown in Fig. 1d. The generated signal at the non-acupoint was roughly one half of the amplitude but its wavelength was just slightly less. Thus, it appears that nonacupoint activation could have a similar but reduced effect in comparison with acupoint activation. The anisotropic indices for the acupoint and non-acupoint in the example given in Fig. 1c, d are 2.46 and $1.47 \mathrm{~g} / \mathrm{cm} \mathrm{s}^{2}$, respectively.
The measured elasticity values were statistically tested using one-way ANOVA.

A series of experiments focusing on ion channels have been designed to uncover the ASW effect of acupuncture. The experimental setup is shown in Fig. 2a. The acupuncture needle was driven by a piezoelectric driver. It produced the ASW that was applied to the tissue-simulating methyl cellulose medium. The wave then propagated to reach the targeted cells seeded at the bottom. Images were taken by MRE to characterize the wavelength, amplitude, and attenuation of the ASW (Fig. 2b). Acoustic pressure distribution in methyl cellulose was simulated by COMSOL Multiphysics (Fig. 2c). Intracellular signals were monitored and recorded by confocal microscopy.

We first used a mouse embryonic fibroblast cell line (NIH 3T3) as a model. A rise in cytosolic $\mathrm{Ca}^{2+}$ was observed following needle driver stimulation. The phase shifts of $\mathrm{Ca}^{2+}$ signals in three individual cells (Fig. 2d) show that the $\mathrm{Ca}^{2+}$ transient first appears (1) at the upper left corner $0.5 \mathrm{~cm}$ from the needle, then spreads towards (2) the center, and finally (3) at the lower right corner. A quantitative analysis of the $\mathrm{Ca}^{2+}$ rise times and peak amplitudes are shown in Fig. 2e, f. The normalized peak amplitude of the $\mathrm{Ca}^{2+}$ rises also reduced gradually from 0.55 at the source of the acoustic wave (1), through 0.3 at point 2 to 0.15 at the final point 3 . The normalized peak amplitude exponentially decays with the distance away from the source of acoustic wave (normalized peak amplitude $\exp (-x / d)$ ), primarily resulting from the ASW attenuation during propagation. Attendant to the peak amplitude attenuation is the significantly increasing latency for stimulated cytosolic $\mathrm{Ca}^{2+}$ rise from 50 through 125 to $325 \mathrm{~s}$. It is noted that the latency and amplitude of the $\mathrm{Ca}^{2+}$ transient could be tuned by the local strength of the waves.

A second cell model was murine microvascular endothelial cell line H5V. H5V cells responded to acupunctureinduced acoustic waves by oscillatory rise in cytosolic $\mathrm{Ca}^{2+}$. Figure $3 \mathrm{a}$ indicates that responses are extremely heterogeneous in frequency, amplitude, and latency due to the spatial dependence of acoustic wave intensities. The average $\mathrm{Ca}^{2+}$ oscillation frequency and peak amplitude decreased modestly as the acoustic wave attenuates with distance (Fig. 3a). In addition, there was a significant change in the latency for $\mathrm{Ca}^{2+}$ rise: $4.98 \pm 1.01 \mathrm{~min}$ at a site $0.5 \mathrm{~cm}$ from the needle driver, $14.40 \pm 5.19 \mathrm{~min}$ at $1 \mathrm{~cm}$, and $32.75 \pm 7.23 \mathrm{~min}$ at $1.5 \mathrm{~cm}$. Similar to NIH 3T3, gating threshold to elicit cytosolic $\mathrm{Ca}^{2+}$ rise in $\mathrm{H} 5 \mathrm{~V}$ was elevated where the acoustic signal amplitude was small (Fig. 3c). A surprising finding is that the $\mathrm{Ca}^{2+}$ oscillation remained at $30 \mathrm{~min}$ or even $1.5 \mathrm{~h}$ after the needle simulation was turned off (Fig. 3b). The latency and memory effects appeared to be in agreement with the long-lasting healing claimed in traditional acupuncture treatment. Fixing the driver voltage 


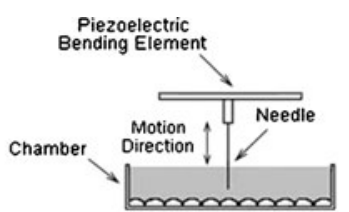

a
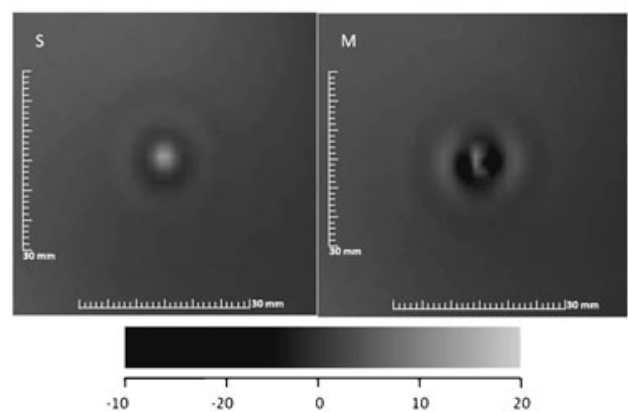

b

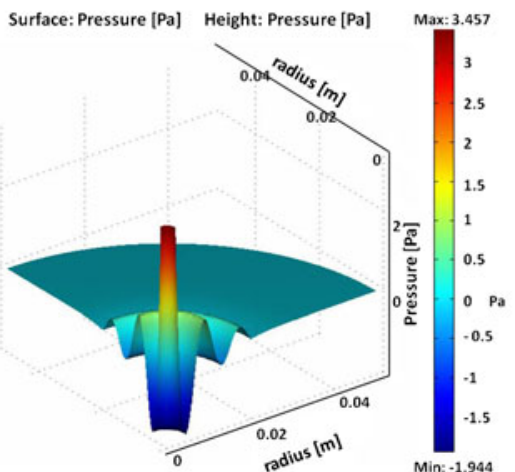

C
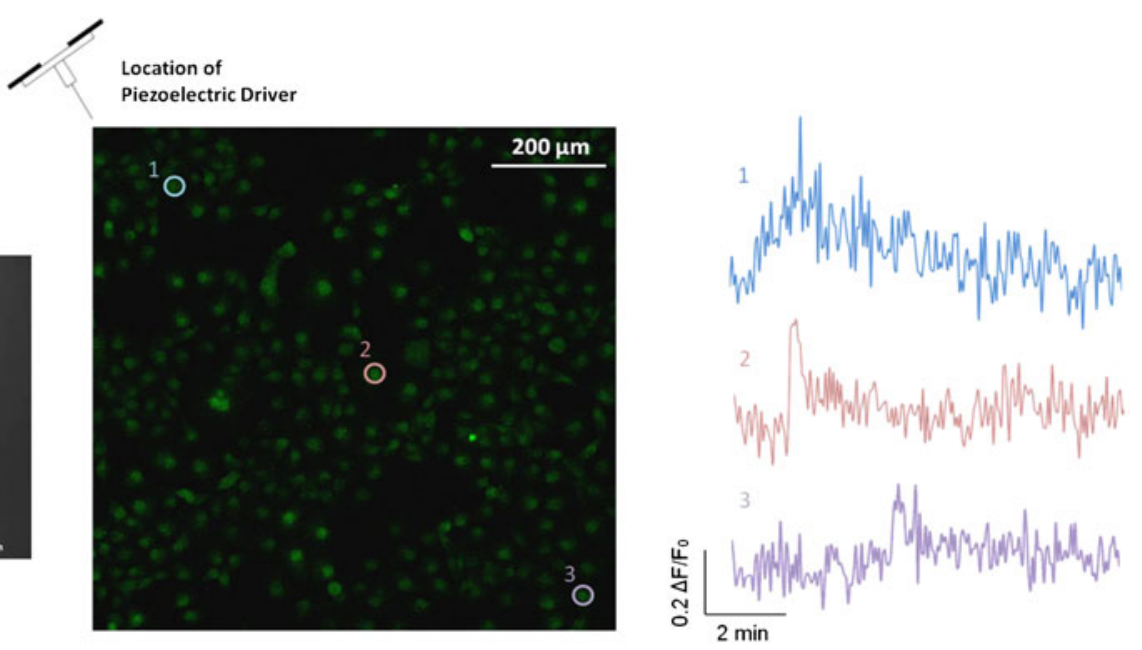

d

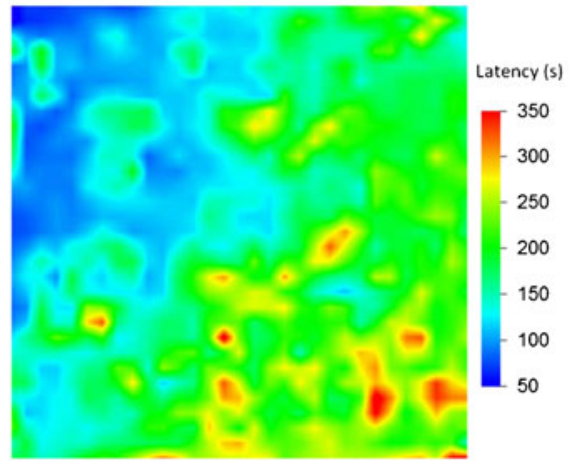

e

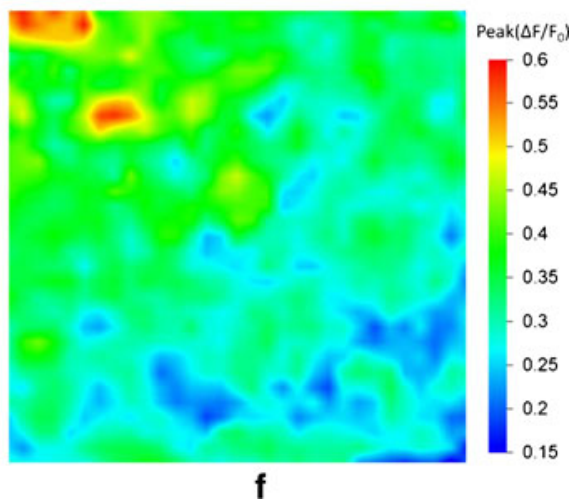

Fig. $2 \mathrm{Ca}^{2+}$ excitation in fibroblast (NIH 3T3) by acupunctureinduced acoustic waves. a Piezoelectric driver to provide vertical motion of the needle inside methyl cellulose solution. b MRE wave images generated by the needle shear wave driver. c Simulated acoustic pressure distribution of methyl cellulose solution. COMSOL Multiphysics 3.4 was used to simulate the coupling of the needle shear wave to methyl cellulose. The maximum acoustic pressure is $3.457 \mathrm{~Pa}$ at the center. d Confocal microscopy image of Calcium Green-1loaded NIH 3 T3 cells under a $\times 10$ objective lens. Peak $\mathrm{Ca}^{2+}$ transient spread from cells at the upper left then propagate to neighboring cells seconds later. e The 3D color-coded map shows the spatial distribution of latency of propagating $\mathrm{Ca}^{2+}$. The latency mesh is taken as the average of six individual experiments. f A 3D contour plot shows the spatial distribution of normalized peak amplitudes of $\mathrm{Ca}^{2+}$ transients. The amplitude mesh is taken as the average of six individual experiments. The product of the peak amplitude square and the latency is calculated to be constant of $\sim 13$ at points $1(0.52 \times 0.52 \times 45=$ $12.2), 2(0.35 \times 0.35 \times 100=12.3)$, and $3(0.23 \times 0.23 \times 230=12.1)$ of the piezoelectric element, the frequency response of the amplitude decreases linearly in Fig. 3d.

$\mathrm{Ca}^{2+}$ activation evoked by acupuncture-induced acoustic waves was also present in cells originating from different tissues including ventricular myocytes and PC-12, as shown in Fig. 4. Since both excitable and non-excitable cells were activated by ASW, it might be a fundamental cellular property. Thus, this study provides new insight with a better understanding of acupuncture's effectiveness. To further understand the cellular basis of acupuncture needling, we have made additional studies of the $\mathrm{Ca}^{2+}$ channels involved in ASW response and found the following: (1) Acupuncture needle stimulation induced $\mathrm{Ca}^{2+}$ excitation in $\mathrm{NIH} 3 \mathrm{~T} 3$ and H5V, but failed to elicit $\mathrm{Ca}^{2+}$ in the cells treated with $\mathrm{Ca}^{2+}-$ free, 5-mM ethylene glycol tetraacetic acid buffer; (2) the application of $100 \mu \mathrm{M} \mathrm{Gd}^{3+}$, a non-specific mechanosensitive $\mathrm{Ca}^{2+}$ channel blocker, completely abolished the channel activity; and (3) 200- $\mu \mathrm{M}$ streptomycin, another blocker for mechanosensitive $\mathrm{Ca}^{2+}$ channels, also inhibited the $\mathrm{Ca}^{2+}$ rise.

In vivo experiments were carried out on 8-week-old C57BL/6N mice. The mouse's hind limb muscle was transfected with GCaMP2 cDNA by electroporation. Two weeks after transfection, the animal was anesthetized, shaved, mounted, and acupuncture needle was inserted into the hind limb muscle at acupoint ST36. $\mathrm{Ca}^{2+}$ level was monitored by two-photon confocal microscopy. ASW induced a robust and significant increase in fluorescence in hind limb muscle fiber at the site of stimulation and the ASW- 


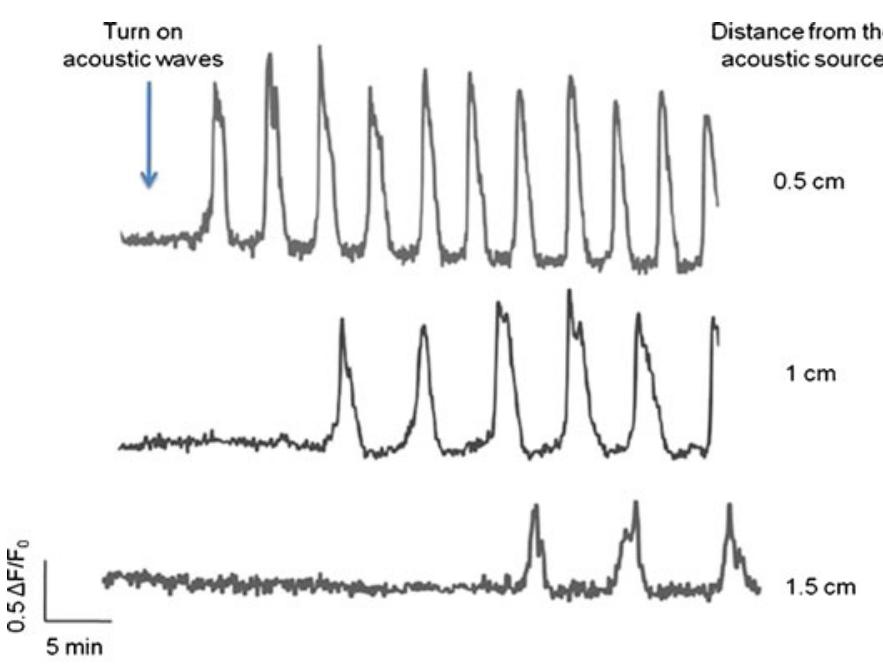

a

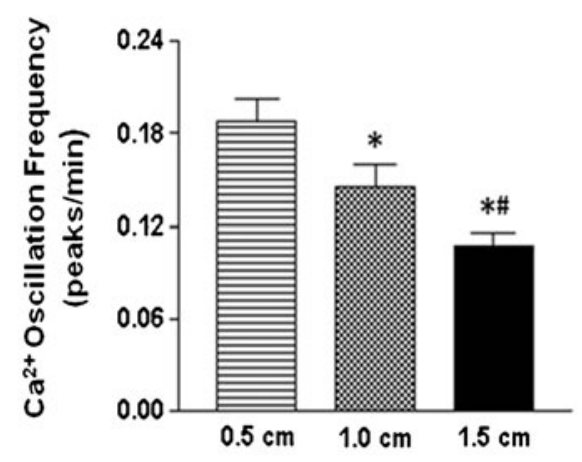

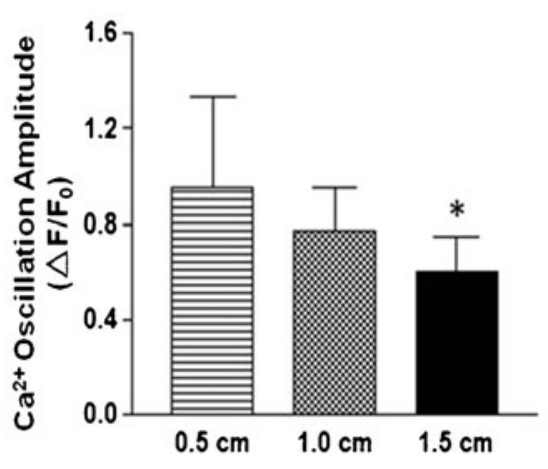

C

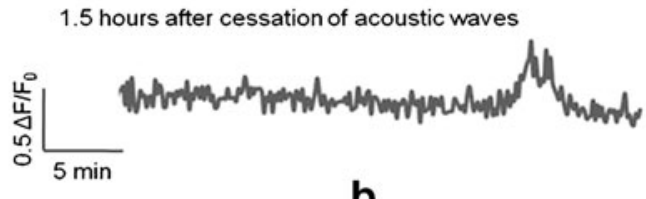

$30 \mathrm{~min}$ after cessation of acoustic waves

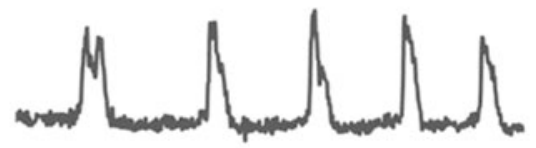

b

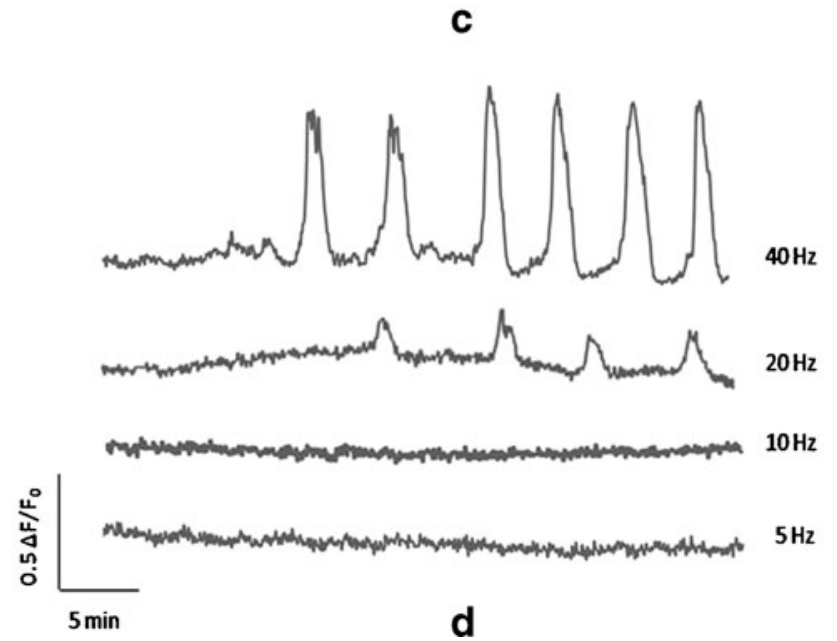

Fig. $3 \mathrm{Ca}^{2+}$ oscillations when endothelial $\mathrm{H} 5 \mathrm{~V}$ cells are exposed to ASW propagating in high-viscosity methyl cellulose solution. a Three typical profiles of single-cell $\mathrm{Ca}^{2+}$ recording. b Memory effects of H5V c Statistical analysis of the dose-dependent effect of single-cell $\mathrm{Ca}^{2+}$ oscillations. The $\mathrm{Ca}^{2+}$ oscillation frequency and peak $\mathrm{Ca}^{2+}$ amplitude decrease as the acoustic wave attenuates with distance $(0.5$,

induced fluorescence signal was subsequently propagated to the adjacent muscle fibers, suggesting an acupuncturestimulated $\mathrm{Ca}^{2+}$ response (Fig. 5a, b). The light emission
1 , and $1.5 \mathrm{~cm}$ from the acoustic source, $n=8 \times 3=24$ ). And the singlecell $\mathrm{Ca}^{2+}$ response delay increases as the acoustic wave diminishes in the medium. ${ }^{*} p<0.05$, significant versus the $0.5-\mathrm{cm}$ group; $\# p<0.05$, significant versus the 1-cm group. d Response of $\mathrm{H} 5 \mathrm{~V}$ subjected to acoustic wave of different frequencies; region of interest around $0.5 \mathrm{~cm}$ away from the needle driver driven by a constant voltage

enhancement is similar to that of $\mathrm{Ca}^{2+}$ uptake into the mitochondria of the mouse's hind limb muscle upon tetanic contraction [18]. 


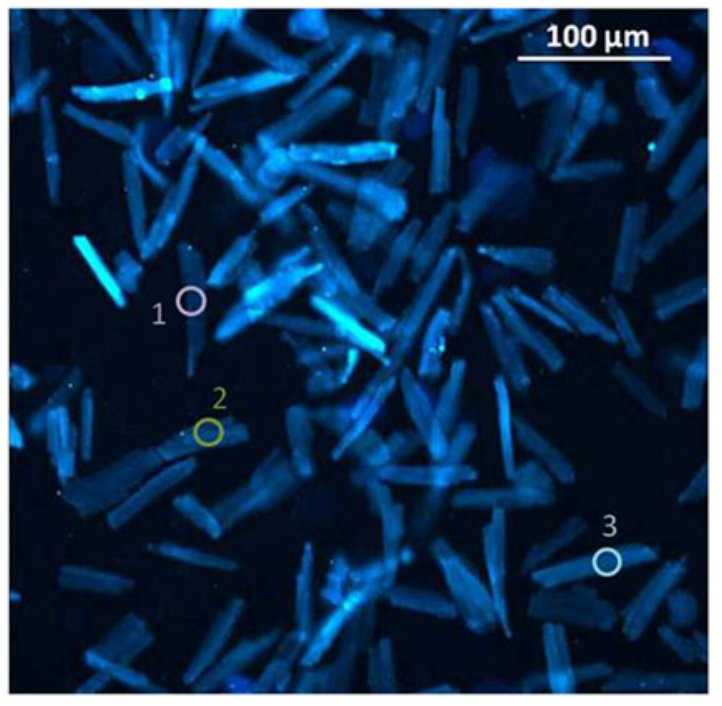

a
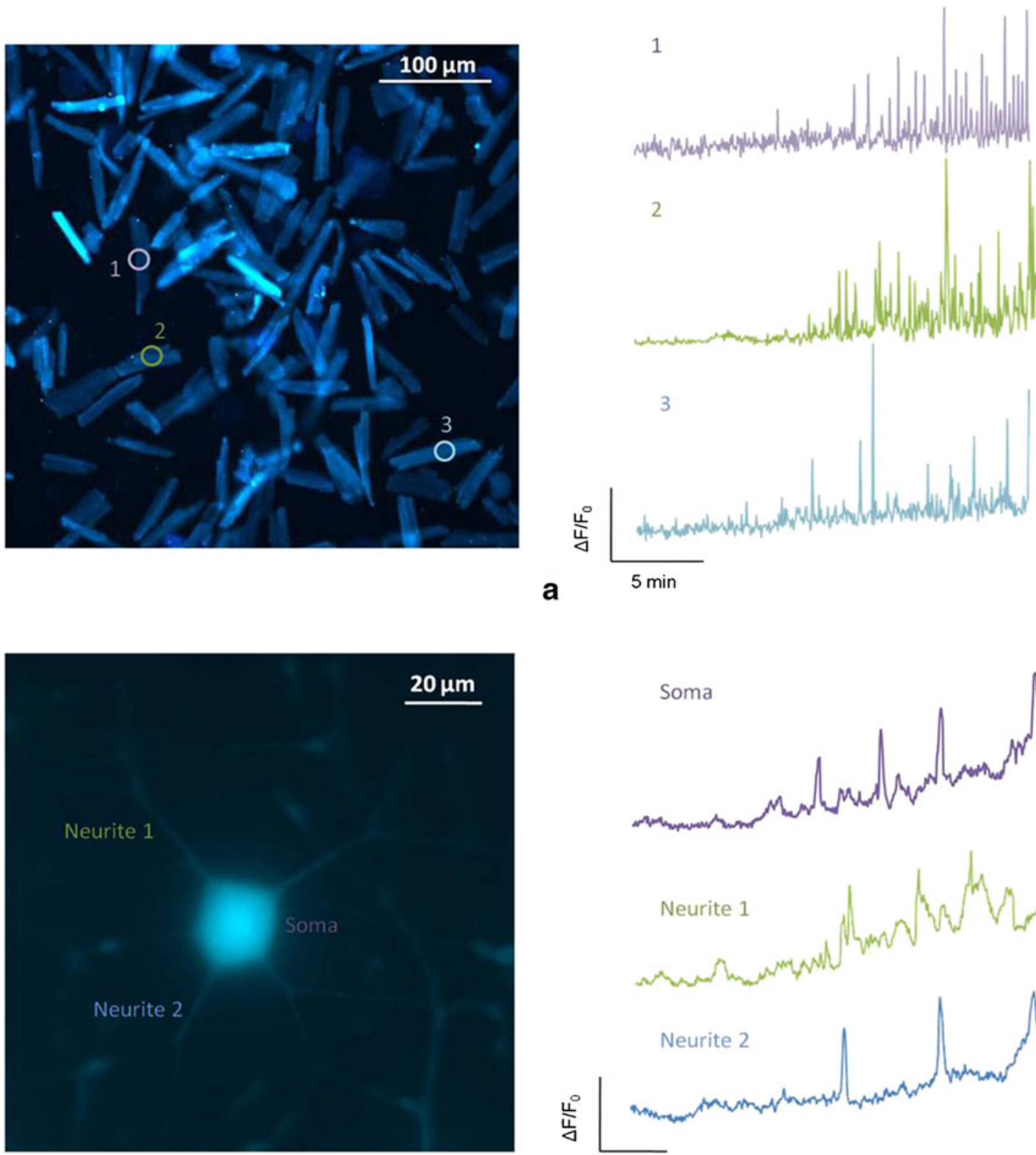

Fig. $4 \mathrm{Ca}^{2+}$ excitation of rat ventricular myocytes and differentiated PC-12 in response to acupuncture-induced acoustic waves. a Confocal microscopy image of Fura-2/AM-loaded ventricular myocytes under a $\times 20$ objective lens. Acoustic waves are turned on within 1 min upon recording. Contraction pulses and increase of $\mathrm{Ca}^{2+}$ transients are

In the second in vivo experiment, acupuncture was initiated by the needle driver described above in the hind limb muscle of the mouse. Plasma $\beta$-endorphin was measured 40 min after the acupuncture treatment by immunoassay. Elevation of $\beta$ endorphin levels was observed (Fig. 5c) along with cytosolic $\mathrm{Ca}^{2+}$ activation in muscle fibers in vivo 40 min after the stimulation was applied. The acupuncture-induced $\beta$ endorphin increase was blocked by the intraperitoneal injection of $\mathrm{Gd}^{3+}$, an inhibitor for mechanosensitive $\mathrm{Ca}^{2+}$

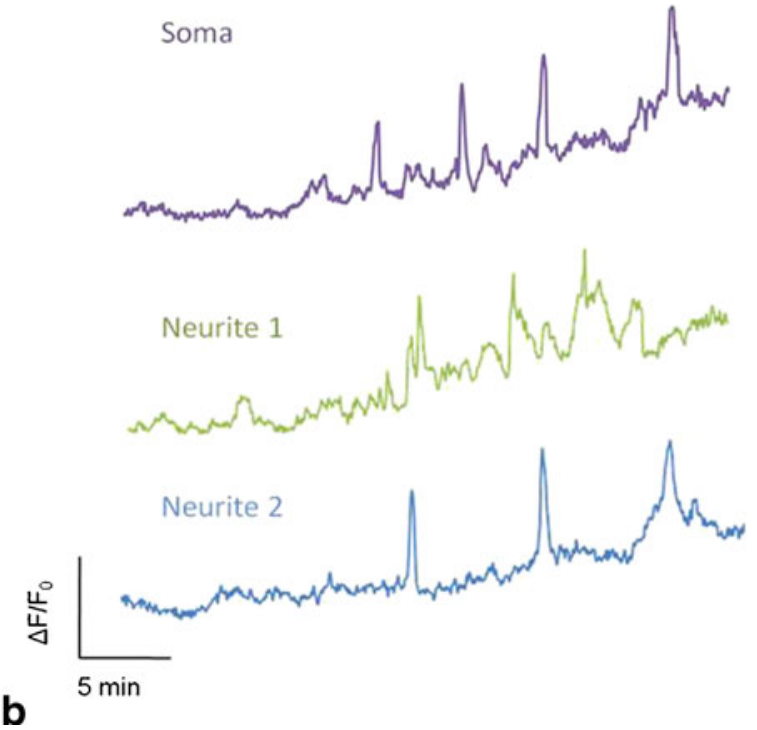

detected after a certain period of delay. $\mathbf{b}$ Confocal microscopy image of Fura-2/AM-loaded differentiated PC- 12 under a $\times 60$ objective lens. $\mathrm{Ca}^{2+}$ oscillations of differentiated PC-12 are detected when exposed to acoustic waves

channels. It is possible that acupuncture-induced $\mathrm{Ca}^{2+}$ signaling may contribute to $\beta$-endorphin release.

\section{Discussion}

In acupuncture, the signal is broadcasted with the wave radiating from the needle like a radio antenna. The muscle, being a fiber bundle, is an anisotropic medium for sound as 
Fig. 5 In vivo $\mathrm{Ca}^{2+}$ signals induced by acupuncture in mouse skeletal muscle. a Transfected muscle fibers exhibited weak GCaMP2 fluorescence in vivo without ASW. b Enhanced brightness in fluorescence signal of GCaMP2 observed under ASW stimulation indicates cytosolic $\mathrm{Ca}^{2+}$ was excited by acupuncture. $\mathbf{c ~ G d ^ { 3 + }}$ completely reversed acupuncture-induced increase in plasma $\beta$-endorphin of the mice. Error bars represent SEM; $n=4 ; * p<0.05$ versus the acupuncture-treated group
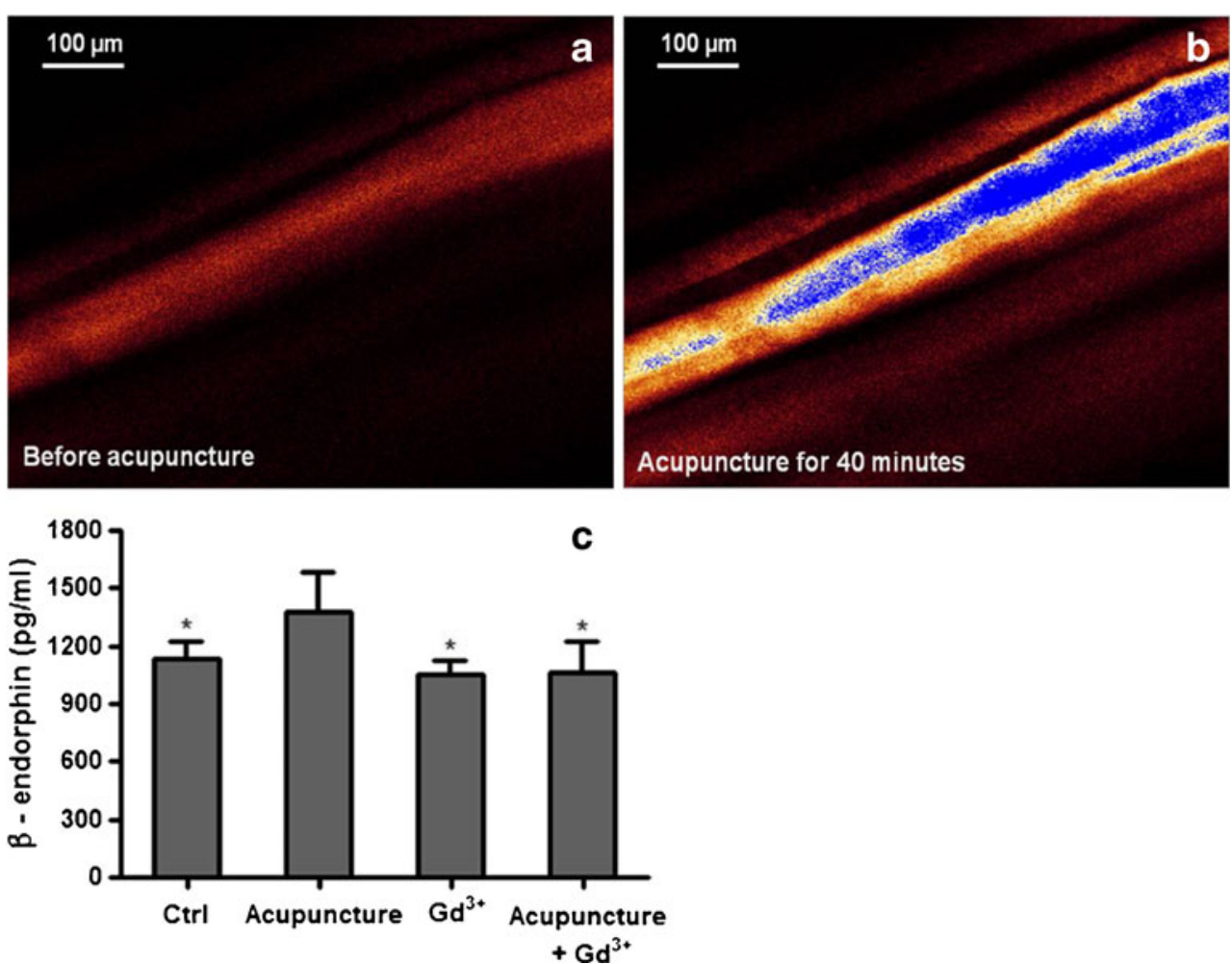

observed in wave propagation through the biceps brachii muscle with a chevron-like pattern [5]. With low absorption coefficient at low frequency, the attenuation is small so that the acoustic wave can easily reach the cells or nerve endings. In addition, the elasticity of the muscle in the relaxed and contracted state can differ by more than a 100fold [6], and an 8-fold increase has been detected in MRE [5]. The insertion of a needle tends to contract a muscle, increasing the anisotropy and reducing the attenuation. These physical properties made the muscle an effective but somewhat leaky acoustic waveguide. As in any waveguide, symmetry is important for wave propagation. In a muscle, the acupoint and non-acupoint did not induce the same tissue displacement. With the same applied voltage to the piezoelectric transducer, the acoustic wave amplitude at the acupoint and the sham point differed by a factor of $\sim 2.5$, as shown in Fig. 1c, d. As pointed out previously, the connective tissue network may play a key role in the transmission of the mechanical needling process of cellular events [12]. The acupoint and sham point, being laterally separated by $1 \mathrm{~cm}$, are likely to engage different collagen fibers, producing different mechanical coupling. Thus, the importance of stress guiding and anatomical distinction between acupoint and sham point cannot be underestimated. This phenomenon would explain a significant contrast in producing the acupuncture signal and its pathway.

Although there was a substantial difference between the responses of the acupoint and sham point, both could produce a measurable ASW that could reach the cell levels. It would also depend on the anatomical location of the points of activation. Our experimental finding is that the coupling or energy transfer at the acupoint and sham point appears to produce the difference in signal amplitude, and the cell latency primarily relates to the attenuated acoustic signal of which the cell is sensitive (Fig. 2). We also discovered that there appears to be a constant value for the product of the peak amplitude square and the latency $\left(A_{\mathrm{o}}{ }^{2} \times\right.$ latency $=$ constant), suggesting a threshold acoustic energy required for activating the cell. Further support for this line of reasoning is evidenced by the experimental observations (Fig. 3d) that the peak amplitude square has an inverse dependence on the applied acoustic signal frequency (between 10 and $80 \mathrm{~Hz}$ ), indicating that the acupuncture is actually independent of the applied signal frequency, but strictly follows the acoustic energy. This would also explain why some of the clinical trials, e.g., in migraine, knee and back pain studies, showed that both methods were effective $[4,7,14,23]$. It is also consistent with the observation that the traditional acupoint activation was, in most cases, better than the sham acupuncture $[4,23]$. In practice, the clinician does not have to hit the bull's eye and deviation from acupoints is acceptable.

As for the mechanotransduction, we observed that the ASW is able to produce cytosolic $\mathrm{Ca}^{2+}$ oscillations in endothelial cells similar to that produced by a $\mathrm{Ca}^{2+}$. mobilizing agonist histamine [9] (Fig. 3a). It is significant 
that there is no involvement of action potential or electrical excitation and that only a change in cytosolic $\mathrm{Ca}^{2+}$ is involved. In the literature, it is widely believed that $\mathrm{Ca}^{2+}$ oscillations can serve as second messenger $[2,22]$. The memory effect seen in Fig. $3 b$ can be explained by having the endoplasmic reticulum as the $\mathrm{Ca}^{2+}$ stores and its buildup and release could produce the regenerative oscillation. This is, however, speculative and requires more study $[8,17]$. In our data, the surprisingly long-lasting $\mathrm{Ca}^{2+}$ oscillations seen after an hour of actuation are testimonies of the power of acupuncture in migrant headache in which healing is now recognized more effective than a chemical drug and yet without side effects.

Another significant point in our study is the result of in vivo two-photon microscopy with ASW. Previously, it was demonstrated that both cytoplasmic and mitochondrial $\mathrm{Ca}^{2+}$ transients can be monitored using the genetically encoded fluorescent calcium indicator protein GCaMP2 [21]. In vivo and under highly physiological conditions, mitochondria in mouse skeletal muscle take up $\mathrm{Ca}^{2+}$ during contraction and rapidly release it during relaxation. The rise in $\mathrm{Ca}^{2+}$ observed during single twitches and tetanic stimulation in live mammalian skeletal muscle is synchronized with the physiological induction of muscle contraction [18]. Instead of inducing contraction by motor nerve stimulation, we have found that the ASW is capable of duplicating the same function. The enhancement of endorphin release and its inhibition by $\mathrm{Gd}^{3+}$ with the in vivo mouse study demonstrated the central role of $\mathrm{Ca}^{2+}$. Without ASW, there was no increase in cytosolic $\mathrm{Ca}^{2+}$ or endorphin. ASW produced an endorphin increase, which was blocked by $\mathrm{Gd}^{3+}$. Thus, it appears that acupuncture may play the role as the trigger making possible pain relief. Different from other modes of mechanical stimuli, ASW is long-range and dynamic. Thus, latency, memory, $\mathrm{Ca}^{2+}$ wave, and accumulative effect can be readily explained in ASW. It should be pointed out that, although the therapeutic effect can be modeled with ASW, our results only suggest a plausible self-consistent mechanism. More experiments are needed to confirm conclusively the effect of analgesia or pain suppression.

\section{Conclusion}

Modern imaging and cell biology techniques have been employed to study the nature of acupuncture. By using a vibrating acupuncture needle with a displacement in the range of $100 \mu \mathrm{m}$, we have been able to demonstrate that the ancient healing technique can be modeled by the ASW. The ASW, being a mechanical energy, is capable of mechanotransduction, stimulating cytosolic $\mathrm{Ca}^{2+}$ rise in both excitable and non-excitable cells, producing $\mathrm{Ca}^{2+}$ oscillations, memory, and giving rise to in vivo calcium fluorescence and endorphin release into blood plasma in mice. The mechanism obeys the wave equations and the laws of cell biology so that acupuncture is unencumbered by the mystery of the celestial lancets and its unfathomable ramifications.

Acknowledgments In memory of Professor Sir Harry Fang, MD, whose counsel, confidence, and unfailing support made this research possible. ESY would also like to thank Dr. Allan Wong for his extraordinary kindness and support during his sojourn in Hong Kong. Generous with his time and ideas, Dr. Peace Cheng (Peking U) made suggestions in cell line studies and helped supervise J. Liang. The assistance of A. Kwan (CUHK) and W. J. Xie (PKU) in cell line experiments is gratefully acknowledged. Early funding was provided by HK Jockey Club and HKU Foundation.

Conflicts of interest There is no conflict of interest of sponsor or authors.

Open Access This article is distributed under the terms of the Creative Commons Attribution Noncommercial License which permits any noncommercial use, distribution, and reproduction in any medium, provided the original author(s) and source are credited.

\section{References}

1. Auld BA (1990) Acoustic fields and waves in solid. Krieger, Melbourne

2. Berridge MJ, Irvine RF (1989) Inositol phosphates and cell signalling. Nature 341:197-205

3. Chan QCC, Li G, Ehman RL, Grimm RC, Li R, Yang ES (2006) Needle shear wave driver for magnetic resonance elastography. Magn Reson Med 55:1175-1179

4. Diener HC et al (2006) Efficacy of acupuncture for the prophylaxis of migraine: a multicentre randomized controlled clinical trial. Lancet Neurol 5:310-316

5. Dresner MA et al (2001) Magnetic resonance elastography of skeletal muscle. J Magn Reson Imaging 13:269-276

6. Duck FA (1990) Physical properties of tissue: a comprehensive reference book. Academic, London

7. Endres HG et al (2007) Acupuncture for the treatment of chronic knee and back pain. Dtsch Arztebl 104:A123-A130

8. Falcke M (2004) Reading the patterns in living cells - the physics of $\mathrm{Ca}^{2+}$ signaling. Adv Phys 53:255-440

9. Jacob R et al (1988) Repetitive spikes in cytoplasmic calcium evoked by histamine in human endothelial cells. Nature 335:40-45

10. Kinsler LE, Frey AR, Coppens AB, Sanders JV (1990) Fundamentals of acoustics. Wiley, New York

11. Kong $J$ et al (2007) Test-retest study of fMRI signal change evoked by electroacupuncture stimulation. NeuroImage 34:1711181

12. Langevin HM et al (2004) Tissue displacements during acupuncture using ultrasound elastography techniques. Ultrasound Med Biol 30:1173-1183

13. Li G et al (2006) An fMRI study of somatosensory-implicated acupuncture points in stable somatosensory stroke patients. J Magn Reson Imaging 24:1018-1024

14. Linde $\mathrm{K}$ et al (2005) Acupuncture for patients with migraine. JAMA 293:2118-2125 
15. Lu GD, Needham J (1980) Celestial lancets: a history and rationale of acupuncture and moxibustion. Cambridge University Press, Cambridge, UK

16. Muthupillai R et al (1995) Magnetic resonance elastography by direct visualization of propagating acoustic strain waves. Science 269(5232):1854-1857

17. Politi A, Gaspers LD, Thomas AP, Hofer T (2006) Models of IP3 and $\mathrm{Ca}^{2+}$ oscillations: frequency encoding and identification of underlying feedback. Biophys J 90:3120-3133

18. Rudolf $\mathrm{R}$ et al (2004) In vivo monitoring of $\mathrm{Ca}^{2+}$ uptake into mitochondria of mouse skeletal muscle during contraction. J Cell Biol 166:527-536
19. Sinkus R et al (2005) Imaging anisotropic and viscous properties of breast tissue by magnetic resonance elastrography. Magn Reson Med 53:273-287

20. Stux G, Berman B, Pomeranz B (2003) Basics of acupuncture. Springer, Berlin

21. Tallini YN et al (2006) Imaging cellular signals in the heart in vivo: cardiac expression of the high-signal $\mathrm{Ca}^{2+}$ indicator GCaMP2. Proc Natl Acad Sci USA 103:4753-4758

22. Tsien RW, Tsien RY (1990) Calcium channels, stores and oscillations. Annu Rev Cell Biol 6:715-7602

23. Witt $C$ et al (2005) Acupuncture in patients with osteoarthritis of the knee: a randomized trial. Lancet 366:136-143 